\title{
ON BURKHOLDER'S BICONVEX-FUNCTION CHARACTERIZATION OF HILBERT SPACES
}

\author{
JINSIK MOK LEE
}

(Communicated by William J. Davis)

\begin{abstract}
Suppose that $\mathbf{X}$ is a real or complex Banach space with norm $|\cdot|$. Then $\mathbf{X}$ is a Hilbert space if and only if

$$
E|x+Y| \geq 1
$$

for all $x \in \mathbf{X}$ and all $\mathbf{X}$-valued Bochner integrable functions $Y$ on the Lebesgue unit interval satisfying $E Y=0$ and $|Y| \geq 1$ a.e. This leads to a simple proof of the biconvex-function characterization due to Burkholder.
\end{abstract}

\section{INTRODUCTION}

Suppose that $\mathbf{X}$ is a real or complex Banach space with norm $|\cdot|$. Then $\mathbf{X}$ is $\zeta$-convex if there is a biconvex function $\zeta: \mathbf{X} \times \mathbf{X} \rightarrow \mathbf{R}$ such that $\zeta(0,0)>0$ and

$$
\zeta(x, y) \leq|x+y| \quad \text { if }|x|=|y|=1 .
$$

Biconvexity means that both $\zeta(\cdot, y)$ and $\zeta(x, \cdot)$ are convex on $\mathbf{X}$ for all $y$ and $x$ in $\mathbf{X}$.

The condition of $\zeta$-convexity, discovered by Burkholder, characterizes Banach spaces with the unconditionality property for martingale differences (UMD); see $[3,6]$. The condition of $\zeta$-convexity also characterizes a class of Banach spaces important in harmonic analysis. Burkholder and McConnell [5] proved that if $\mathbf{X}$ is $\zeta$-convex, then the Hilbert transform, defined by

$$
H f(x)=\lim _{\varepsilon \downarrow 0} \frac{1}{\pi} \int_{|y|>\varepsilon} \frac{f(x-y)}{y} d y,
$$

is a bounded operator on the Lebesgue-Bochner space $L^{p}(\mathbf{R}, \mathbf{X})$ for $1<p<$ $\infty$, and obtained similar results for more general singular integral operators. Later Bourgain [2] proved the converse: If the Hilbert transform is a bounded operator on $L^{p}(\mathbf{R}, \mathbf{X})$, then $\mathbf{X}$ is $\zeta$-convex. (See [7] for background information on the Bochner integral.)

If $\zeta: \mathbf{X} \times \mathbf{X} \rightarrow \mathbf{R}$ is a function satisfying (1), then

$$
\zeta(0,0) \leq 1 .
$$

Received by the editors October 20, 1991.

1991 Mathematics Subject Classification. Primary 46C15; Secondary 46B09.

Key words and phrases. Biconvexity, $\zeta$-convexity, Bochner integrable functions. 
To see this, take $x$ in $\mathbf{X}$ with $|x|=1$. Then by biconvexity and (1),

$$
\zeta(0,0) \leq \frac{1}{4}\{\zeta(x, x)+\zeta(x,-x)+\zeta(-x, x)+\zeta(-x,-x)\} \leq|x|=1 .
$$

If $\mathbf{H}$ is a Hilbert space, there is a biconvex function $\zeta$ on $\mathbf{H} \times \mathbf{H}$ that attains the upper bound in (2). Let

$$
\zeta(x, y)=1+(x, y)
$$

where $(\cdot, \cdot)$ denotes the real part of the inner product of $x$ and $y$. Then $\zeta$ is biconvex and $\zeta(0,0)=1$. Furthermore, $\zeta$ satisfies (1):

$$
\begin{aligned}
\zeta(x, y)^{2} & \leq 1+2(x, y)+|x|^{2}|y|^{2} \\
& =|x+y|^{2}+\left(1-|x|^{2}\right)\left(1-|y|^{2}\right) .
\end{aligned}
$$

As Burkholder observed (see [4, 6]), the converse holds.

Theorem 1. Suppose that $\mathbf{X}$ is a Banach space. If there is a biconvex function $\zeta: \mathbf{X} \times \mathbf{X} \rightarrow \mathbf{R}$ such that $\zeta(0,0)=1$ and $(1)$ is satisfied, then $\mathbf{X}$ is a Hilbert space.

The proof given by Burkholder is geometrical. He reduces it in several steps to the parallelogram identity of Jordan and von Neumann. To prove Theorem 1 from a different perspective we shall consider a seemingly unrelated problem.

\section{THE MAIN THEOREM}

Let $x$ be a point in the Banach space $\mathbf{X}$ and $Y$ an $\mathbf{X}$-valued Bochner integrable function on the Lebesgue unit interval. Denote by $E Y$ the integral of $Y$ on $[0,1)$. Assume that $E Y=0$ and $|Y| \geq 1$ a.e. Then the question is: How small can $E|x+Y|$ be?

The following lemma provides a lower bound.

Lemma 2. If $x \in \mathbf{X}$ and $Y$ is an $\mathbf{X}$-valued Bochner integrable function on the Lebesgue unit interval satisfying $E Y=0$ and $|Y| \geq 1$ a.e., then

$$
E|x+Y| \geq \zeta(0,0)
$$

for all biconvex functions $\zeta$ on $\mathbf{X} \times \mathbf{X}$ satisfying (1).

This lemma follows from Lemma 8.1 of [6], but we shall give a direct proof here.

I. If there is a biconvex function $u$ on $\{(x, y) \in \mathbf{X} \times \mathbf{X}:|x| \vee|y| \leq 1\}$ satisfying (1), then there is a biconvex function $\zeta$ on $\mathbf{X} \times \mathbf{X}$ such that $\zeta(0,0) \geq$ $u(0,0)$ and

$$
\zeta(x, y) \leq|x+y| \quad \text { if }|x| \vee|y| \geq 1 .
$$

II. If $\zeta$ is a biconvex function on $\mathbf{X} \times \mathbf{X}$ satisfying (5), then for all $x, y$, $x^{\prime}, y^{\prime}$ in $\mathbf{X}$,

$$
\left|\zeta(x, y)-\zeta\left(x^{\prime}, y^{\prime}\right)\right| \leq\left|x-x^{\prime}\right|+\left|y-y^{\prime}\right|
$$

so $\zeta$ is continuous.

See [6] for these and related results.

Proof of Lemma 2. Take $x$ and $Y$ as in Lemma 2, and let $\zeta$ be a biconvex function satisfying (1). By $I$, we can assume that $\zeta$ satisfies (5). Replacing $\zeta$ 
by the mapping $(x, y) \mapsto \zeta(x, y) \vee \zeta(-x,-y)$, if necessary, we can assume that $\zeta$ satisfies the symmetry condition

$$
\zeta(x, y)=\zeta(-x,-y)
$$

Since $|Y| \geq 1$ a.e., property (5) and Jensen's inequality applied to the continuous function $\zeta(x, \cdot)$ yield

$$
E|x+Y| \geq E \zeta(x, Y) \geq \zeta(x, E Y)=\zeta(x, 0) .
$$

From $(6)$ and the convexity of $\zeta(\cdot, 0)$, it follows that

$$
\zeta(x, 0)=\frac{1}{2}\{\zeta(x, 0)+\zeta(-x, 0)\} \geq \zeta(0,0),
$$

which completes the proof of Lemma 2.

In particular, if $\mathbf{X}$ is a Hilbert space, then Lemma 2 applied to the function $\zeta$ defined in (3) gives $E|x+Y| \geq 1$. A natural question is: Does $E|x+Y| \geq 1$ characterize Hilbert space?

Theorem 3. Suppose that $\mathbf{X}$ is a Banach space. If

$$
E|x+Y| \geq 1
$$

for all $x \in \mathbf{X}$ and all $\mathbf{X}$-valued Bochner integrable functions $Y$ on the Lebesgue unit interval satisfying $E Y=0$ and $|Y| \geq 1$ a.e., then $\mathbf{X}$ is a Hilbert space.

\section{PROOFS OF THE THEOREMS}

In our proofs, we can assume that $\mathbf{X}$ is a Banach space over the real field. We need the following two lemmas from the theory of convex bodies. Lemma 4 is a well-known geometric characterization of Hilbert spaces; see [8, p. 144] for the proof. Lemma 5 is due to Loewner; see [1] or [8, p. 139].

Lemma 4. Suppose that $\mathbf{X}$ is a two-dimensional real Banach space. Then the norm of $\mathbf{X}$ is generated by an inner product if and only if the unit sphere of $\mathbf{X}$ is an ellipse.

Lemma 5. If $C$ is a symmetric (about the origin) closed convex curve in the plane, then there exists a unique ellipse of maximal area inscribed in $C$. The maximal inscribed ellipse touches $C$ in at least four points which are symmetric pairwise.

Proof of Theorem 3. Suppose, on the contrary, that $\mathbf{X}$ is not a Hilbert space. We shall find $x \in \mathbf{X}$ and an $\mathbf{X}$-valued simple function $Y$ so that $E Y=0$, $|Y| \geq 1$ everywhere, but $E|x+Y|<1$.

We can assume, without loss of generality, that the dimension of $\mathbf{X}$ is equal to two. Denote the norm of $\mathbf{X}$ by $|\cdot|$. Let $S_{X}$ be the unit sphere of $\mathbf{X}$ with respect to $|\cdot|$. Then, by Lemma 5 , there is an ellipse $S_{0}$ of maximal area inscribed in $S_{X}$ with at least four distinct contact points which are symmetric pairwise. Denote by $\|\cdot\|$ the norm induced by $S_{0}$. After some affine transformations, we can assume that $S_{0}$ is the unit circle. Let $\pm A$ and $\pm C$ denote four contact points with no contact points in the interior of the arc $\widehat{A C}$. The existence of such points is assured by Lemma 4.

Let $\theta=\frac{1}{2} \angle A O C$, one half of the angle determined by the line segments $\overline{O A}$ and $\overline{O C}$. Here $O$ denotes the origin of $\mathbf{X}$. By taking a rotation, if necessary, 
we can assume that $0<2 \theta \leq \pi / 2, A=(1,0)$, and $C=(\cos 2 \theta, \sin 2 \theta)$. Let $D=s(\cos \theta, \sin \theta)$, where $s$ is a positive number satisfying $|s(\cos \theta, \sin \theta)|=$ 1. Accordingly, $s>1$.

Let, for $t$ in an interval $(-s, s)$,

$$
x(t)=-t(\cos \theta, \sin \theta) .
$$

Let $Y:[0,1) \rightarrow \mathbf{X}$ be a simple function defined by

$$
Y=A I_{[0, p)}+C I_{[p, 2 p)}-D I_{[2 p, 1)}
$$

where $p=s / 2(s+\cos \theta)$ and $I_{[a, b)}$ denotes the indicator function of the interval $[a, b)$. Then it is easy to see that $E Y=0,|Y|=1$ everywhere on $[0,1)$, and

$$
\begin{aligned}
x(t)+Y= & (1-t \cos \theta,-t \sin \theta) I_{[0, p)} \\
& +(\cos 2 \theta-t \cos \theta, \sin 2 \theta-t \sin \theta) I_{[p, 2 p)} \\
& -(s+t)(\cos \theta, \sin \theta) I_{[2 p, 1)} .
\end{aligned}
$$

Let $f$ and $g$ be functions defined on an interval $(-s, s)$ by

$$
\begin{aligned}
f(t)= & E|x(t)+Y| \\
= & p|(1-t \cos \theta,-t \sin \theta)|+p|(\cos 2 \theta-t \cos \theta, \sin 2 \theta-t \sin \theta)| \\
& +(1-2 p) \frac{s+t}{s}, \\
g(t)= & p\|(1-t \cos \theta,-t \sin \theta)\|+p\|(\cos 2 \theta-t \cos \theta, \sin 2 \theta-t \sin \theta)\| \\
& +(1-2 p) \frac{s+t}{s} .
\end{aligned}
$$

Then, for $t$ in $(-s, s)$,

$$
\begin{aligned}
& f(t) \leq g(t) \quad \text { with } f(0)=g(0)=1 \\
& g(t)=2 p\left(1-2 t \cos \theta+t^{2}\right)^{1 / 2}+(1-2 p) \frac{t+s}{s} \\
& g^{\prime}(t)=2 p \frac{t-\cos \theta}{\left(1-2 t \cos \theta+t^{2}\right)^{1 / 2}}+\frac{1-2 p}{s} .
\end{aligned}
$$

In particular,

$$
g^{\prime}(0)=\frac{\left(1-s^{2}\right) \cos \theta}{s(s+\cos \theta)}<0 \quad \text { since } s>1 \text { and } \frac{\pi}{4} \geq \theta>0 .
$$

Since $g^{\prime}(0)<0$, we obtain $f(t) \leq g(t)<1$ for a small positive number $t$. Let $x=x(t)$ for this $t$. Then $E|x+Y|<1$ where $Y$, given by (7), satisfies $E Y=0$ and $|Y| \geq 1$ everywhere. This completes the proof of Theorem 3 .

Proof of Theorem 1. Suppose that $\mathbf{X}$ is not a Hilbert space. Let $\zeta$ be a biconvex function on $\mathbf{X} \times \mathbf{X}$ satisfying (1). Then by Theorem 3, there exist a point $x$ in $\mathbf{X}$ and a simple function $Y$ with values in $\mathbf{X}$ such that $|Y| \geq 1$ a.e., $E Y=0$, but $E|x+Y|<1$. Therefore, by $(4), \zeta(0,0)$ is less than one. This completes the proof of Theorem 1 .

\section{ACKNOWLEDGMENT}

This paper is part of the Ph.D. work of the author under the direction of Professor Donald L. Burkholder. The author wishes to thank him for his kind guidance and valuable suggestions throughout this work. 


\section{REFERENCES}

1. D. Amir, Characterizations of inner product spaces, Oper. Theory: Adv. Appl., vol. 20, Birkhäuser Verlag, Boston, MA, 1986.

2. J. Bourgain, Some remarks on Banach spaces in which martingale difference sequences are unconditional, Ark. Mat. 21 (1983), 163-168.

3. D. L. Burkholder, A geometrical characterization of Banach spaces in which martingale difference sequences are unconditional, Ann. Probab. 9 (1981), 997-1011.

4. __ Martingale transforms and the geometry of Banach spaces, Proc. Third Internat. Conf. on Probability in Banach Spaces (Tufts University, 1980), Lecture Notes in Math., vol. 860, Springer Verlag, Berlin and New York, 1981, pp. 35-50.

5. __, A geometric condition that implies the existence of certain singular integrals of Banachspace-valued functions, Conference on Harmonic Analysis in Honor of Antoni Zygmund (Chicago, 1981) (William Beckner, Alberto P. Calderón, Robert Fefferman, and Peter W. Jones, eds.), Wadsworth, Belmont, CA, 1983, pp. 270-286.

6. _ Martingales and Fourier analysis in Banach spaces, C.I.M.E. Lectures (Varenna (Como), Italy, 1985), Lecture Notes in Math., vol. 1206, Springer-Verlag, Berlin and New York, 1986, pp. 61-108.

7. J. Diestel and J. J. Uhl, Vector measures, Math. Surveys Monographs, vol. 15, Amer. Math. Soc., Providence, RI, 1977.

8. V. I. Istrăţescu, Inner product structures, Reidel, Boston, MA, 1987.

9. T. R. McConnell, On Fourier multiplier transformations of Banach-valued functions, Trans. Amer. Math. Soc. 285 (1984), 739-757.

Department of Mathematics, University of Illinois, Urbana, Illinois 61801

Current address: Department of Mathematics, Sungwha University, 381-7 Samgyong-Dong, Chonan, Choongnam, Republic of Korea 